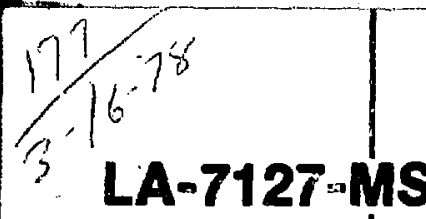

Informal Report
45.927

UC-20g

issu:ad: February 1978

\title{
A Simple Measurement of Line Density
}

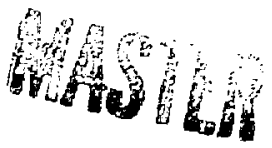

W. T. Armstrong*

R. E. Siemon

"Associated Western Universities Graduate Participant. University of Colorado, Boulder, co $8030 \%$ 


\title{
A SIMPLE MEASUREMENT OF LINE DENSITY
}

by

W. T. Armstrong and $\mathrm{F}$. E. Siemon

\begin{abstract}
Energy line density, $\mathbf{E}_{l}=\int(\mathrm{F} / 2) \mathrm{rk}\left(\mathrm{T}_{\mathrm{0}}+\mathrm{T}_{1}\right) \mathrm{dA}$, has proved to be one of the easiest (and most critical) messurements made on the Scylla IV-P, 5-m linear theta pinch. A quantitative evaluation of end-stoppering techniques is made available by extracting the line energy containment time, $\tau_{E_{\ell}}$, from the time history of $\mathbf{E}_{\ell}$. To model $\mathbf{E}_{\ell}(t)$ properly and, hence, determine the details of energy loss mechanisms (streaming and thermal conduction) cequires separate measurements of $n$ and $T$. A simple measurement of line density, $\mathbf{n}_{\ell}=\int \mathrm{ndA}$, is proposed, in which a single laser beam is passed repeatedly through the plasma colamn along different chords. A discussion is presented of the measurement accuracy as a function of the number of passes.
\end{abstract}

At present, energy line density is calculated from two directly measured quantities: $\Delta \phi$, the excluded flur and $B_{o}$, the vacuum magnetic field; that is,

$E_{\ell}=\frac{F}{2} \frac{B_{0}}{4 \pi} \Delta \phi$,

where $F$ is the number of degrees of freedorn. In terms of plasma parameters, $\mathbf{E}_{\boldsymbol{\ell}}$ is given above as

$E_{\ell}=\frac{F}{2}\{n x T d A$.

Assuming $\mathrm{T}$ is constant over the plasma radius yields

$$
\begin{aligned}
\varepsilon_{\ell} & =\frac{F}{2} k T \_n d A \\
& =\frac{F}{2} n_{\ell} k T .
\end{aligned}
$$

A separate measurement of either $n_{\ell}$ or $T$ determines the relative contribution of particle loss and thermal loss to $\tau_{E_{\ell}}$.

Through neutron production and Thomson scattering measurements, $T_{1}$ and $T_{3}$ can be inferred. It is desirable, however, to measure $n_{\ell}$ independently, as well. Present side-on interferometric techniques ${ }^{2}$ give the integrated chord density, $n_{\mathrm{ch}}$,

$$
n_{c h}=\int_{r=0} n(r, s) d s,
$$

where $s$ is a path along the line of sight and $r$ is the transverse position of tise chord. To some degree, $n_{c h}$ is a desirable measurement by itself. In the low $\beta$ approximation, $a / r_{\phi}=(2 / \beta)^{1 / 2}$ (Ref. 2). With this approximation, a Gaussian profile and $a / r_{\phi} \simeq 1.75 \pm$ $15 \%$ for $0.8>\beta_{A}>0.5$, one may write the approximete expression 
$\varepsilon_{2}=\frac{F}{2}\left[\left(2 \pi / B_{0}\right) k T \int_{r=0}^{n d s}\right]^{2}$,

where $a$ is the mean plasma radius and $r_{\phi}$ is the excluded flux redius $r_{\phi}=\left(\Delta \phi / \pi B_{0}\right)^{1 / 2}$. Alternatively, side-on holographic interferometry gives $n_{c h}$ for all

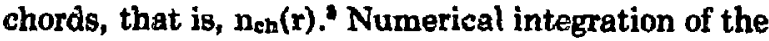
fringe-shift profile results in $\mathbf{n}_{\ell}$. However, only one exposure typically is available during the plasma discharge. A simplified data reduction method is also preferred.

We propose the use of muliple passes through the plasma of a single coherent beam, as illustrated in Fig. 1, which gives an effective measuremant

$$
\begin{aligned}
n_{\ell} & =\int n(r, s) d r d s \\
& \approx \sum_{j} \Delta r_{j} \Gamma_{n}\left(r_{j}, s\right) d s .
\end{aligned}
$$

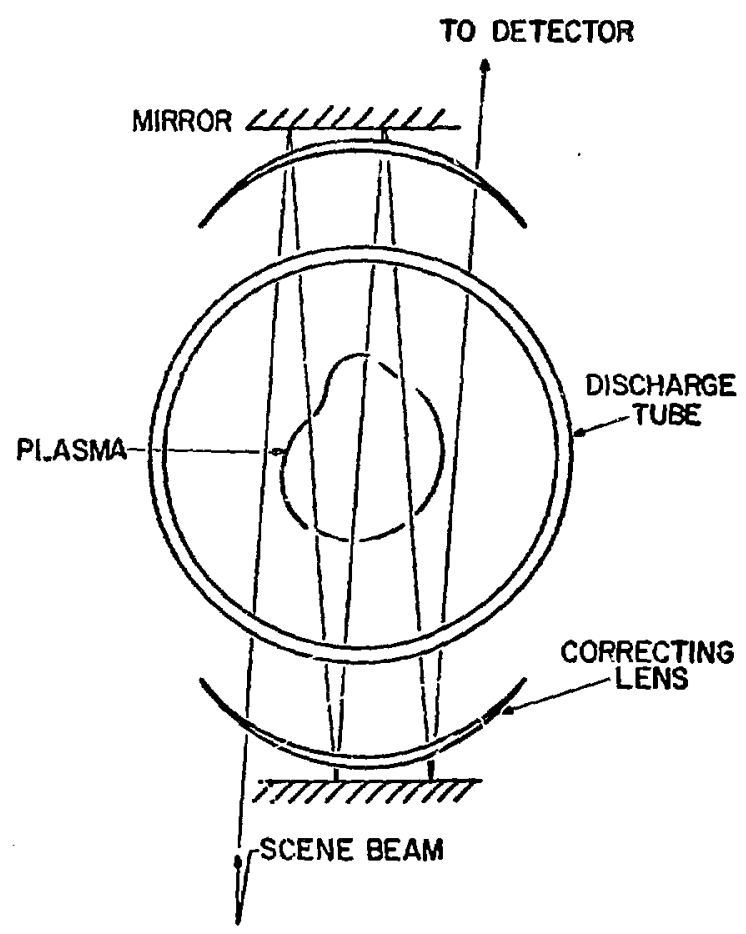

Fig. 1.

Multipass interferometric system for determination of the line density, $n_{l}=\int n d A$.
The result is a single, time-dependent signal, capable of simple recording and independent of plasma shape assumptions. The limitations of this technique are (1) plasma distortions smaller than $\Delta r$ and (2) degradation of beam quality caused by roultiple passes through the discharse tube. At least 10 prsses, spaced over $2 \mathrm{~cm}$, are believed technically possible. This may require a more elaborate window system than that shown in Fig. 1 (for example, a polished tube section or flat windows). Furthermore, a quadrature interferometric system would simplify interpretation of the interferometric signal."

A minimum, required number of passes can be estimated by comparing $n_{\ell}$, calculeted from Eq. (1), to the exact $n_{l}$ for a Gaussian profile. In Fig. 2, the calculated $n_{\ell}$ is plotted as a function of the number of passes. The passes are equally divided across a discharge tube vith a diameter equal to $10 \mathrm{a}$. The exact $n_{\ell}$ is $\pi n_{A} a^{2}$. Figure 2 shows that an odd number of passes overestimates $n_{l}$ and an even number of passes underestimates $n_{l}$ for fewer than 7 passes. The symmetry of the passes (oold or even) with respect to the plasma axis would change as the plasma column moved. Thus, the even and odd curves in Fig. 2 represent bounds of error for varying positions of the plasma column.

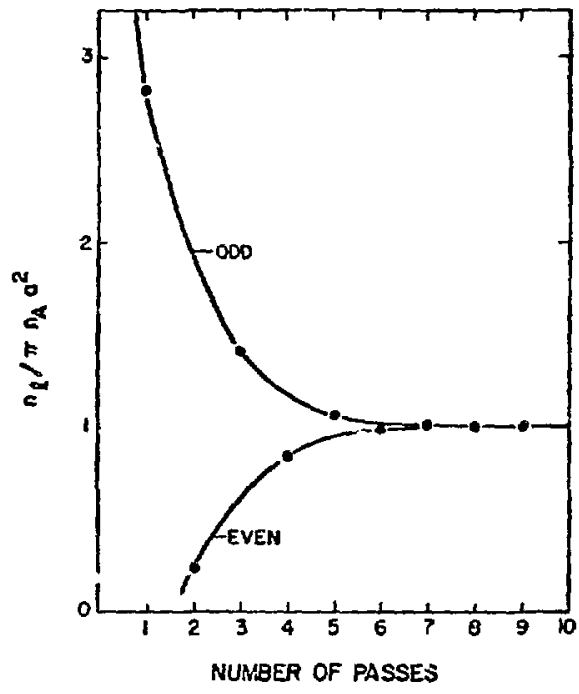

Fig. 2.

Line density $n_{l}$, as calculated from multiple passes, vs number of passes for a Gaussian density profile. 
Convergence to the exact $n_{\ell}$ occurs at $\sim 7$ passes. Faster convergence would be expected if the passes were divided over a length smaller than the "tube diameter" of $10 \mathrm{a}$. Convergence near 7 passes is an indication of the sampling vidth becoming comparable to the plasma widith. To measure $n_{\ell}$ accurately for plasma columns with radial distortions < a, it is advisable to use the maximum number of passes technically possible. This analysis shows that a minimum of 7 passes is a reasonable requirement.

\section{REFERENCES}

1. F. C. Jahoda and G. A. Sawyer, Optical Refractivity of Plasmas, Methods of Experimental Physics,
R. H. Lovberg and H. R. Griem, Eds. (Acaden Press, Inc., New York, 1971), Vol. 9, Part B, pp. 48.

2. Carl Ekdahl, Los Alamos Scientific Laboratol unpublished data, March 1977.

3. R. Kristal, "Pulsed HF Laser Holograph Interferometry," Appl. Opt. 14, 628 (1975).

4. C. J. Buchenauer and A. R. Jacobso "Quadrature Interferometer for Plasma Densi Measurements," Rev. Sci. Instrum. 48, 769 (1977) 